\title{
A Vision-based Navigation System of Mobile Tracking Robot
}

\author{
Jie Wu, Václav Snášel \\ Dept. Computer Science, FEECS \\ VŠB - Technical University of Ostrava \\ Ostrava, Czech Republic \\ defermat2008@hotmail.com, vaclav.snasel@vsb.cz
}

\author{
Ajith Abraham \\ Machine Intelligence Research Labs, MIR Labs \\ http://www.mirlabs.org \\ Washington, USA \\ ajith.abraham@ieee.org
}

\begin{abstract}
Based on the study of developments in many fields of computer vision, a novel computer vision navigation system for mobile tracking robot is presented. According to the primary functions of this kind of robot, three irrelevant technologies, pattern recognition, binocular vision and motion estimation, make up of the basic technologies of our robot. The non-negative matrix factorization (NMF) algorithm is applied to detect the target. The application method of NMF in our robot is demonstrated. Interesting observations on distance measurement and motion capture are discussed in detail. The reasons resulting in error of distance measurement are analyzed. According to the models and formulas of distance measurement error, the error type could be found, which is helpful to decrease the distance error. Based on the diamond search (DS) technology applied in MPEG4, an improved DS algorithm is developed to meet the special requirement of mobile tracking robot.
\end{abstract}

Index Terms - mobile robot, navigation, computer vision, nonnegative matrix factorization, binocular vision, motion estimation.

\section{INTRODUCTION}

A main aim of robotics research is to gain knowledge about the nature of intelligence, to make computers more useful to human. Creating more intelligent computers will make robots more useful to people. Mobile tracking robot can offer some attractive home services closely related to our life. It will be the most useful partner in our life in future. In our project, we try to develop a kind of mass producible, low-cost autonomous mobile tracking robot.

Indubitably, vision navigation system is one of the most important systems in mobile robot. Computer vision for mobile robot navigation became a hot subject since the end of 1970s. It has achieved great progress in the field of computer vision in recent several decades. Many interesting technologies were applied in this field continuously. Most research work on mobile robot vision navigation is focused on map building, self-localization, path planning, and obstacle avoidance.

G. N. DeSouza et al. [1] investigated the developments of twenty years in the field of vision for mobile robot navigation. The developments can be divided into indoor navigation and outdoor navigation.

For the indoor navigation, it includes three broad groups: map-based, map-building-based and mapless navigation. Mapbased navigation systems depend on user-created geometric models or topological maps of the environment. Map-building- based navigation systems use sensors to construct their own geometric or topological models of the environment and then use these models for navigation. Mapless navigation systems use no explicit representation at all about the space in which navigation is to take place, but other resort to recognize objects found in the environment or to track those objects by generating motions based on visual observations. The problem of robotic mapping is that of acquiring a spatial model of a robot's environment. It is a process for robot to perceive the outside world. Sebastian Thrun [2] surveyed major algorithms of robotic mapping, including Kalman filter techniques, approaches based on Dempster's expectation maximization algorithm, occupancy grid techniques, and so on.

For the outdoor navigation, which usually involves obstacleavoidance, land-mark detection, map-building or updating, and position estimation, it can be divided into structured and unstructured environments according to the level of structure of the environment. In general, outdoor navigation in structured environments requires some sort of road-following; while outdoor navigation in unstructured environments, which doesn't require regular properties used to be perceived or tracked for navigation, can make use of at most a generic characterization of the possible obstacles in the environment.

Each sort of those methods mentioned above has its own technical characteristics. The central computations involved in map-based navigation can be divided into four steps: acquire sensory information, detect landmarks, establish matches between observation and expectation and calculate position. The map-building-based approaches try to explore the environment and build an internal representation of it for the robot, such as 3D coordinates, occupancy grid or metrictopological representation, etc. Mapless navigation does not need map, in which the robot motions are determined by observing and extracting relevant information of environment such as walls, desks, doorways, etc. Optical flow-based and appearance-based are two prominent techniques used in maples navigation. Outdoor navigation is often executed by cars or wheeled vehicles. The road-following for outdoor navigation in structured environments means an ability to recognize the lines that separate the lanes or separate the road from the berm, the texture of the road surface, and the adjoining surfaces, etc. In unstructured outdoor navigation, those techniques include 
external camera observation, far-point landmark triangulation, global positioning, etc.

Human eyes and vision navigation system take on similar fundamentality in human and mobile robot, respectively. From the perspective of bionics, the researches on human eyes are referential and significant to the development of robotic vision system. With the important functions, such as binocular parallax, motion parallax, accommodation and convergence, human eyes can extract depth cue out of scene, which finally results in the ability of depth perception. Similarly, if the robotic vision system performs similar functions to those of human eyes, the robot will be more intelligent.

By analyzing those functions of human eyes more carefully, it can be found that there are three processes which are primary and essential to robot: target recognition, distance estimation and motion capture. Target recognition, or object recognition, can tell robot what the target is. Distance estimation can describe the environment; tell robot where the target is and where robot itself is, which actually means some certain kind of stereo vision capability. Motion capture can tell robot how to follow the target. Based on these three primary processes, the mobile tracking robot can possess some important capabilities.

In this paper, we present an approach of computer vision navigation for mobile tracking robot. We try to use cheap cameras and simple algorithm to realize the functions needed by mobile tracking robot. The main process is summarized as follows.

Step 1: Target Recognition. The features of target are abstracted from images taken by the vision system of the mobile robot. In this step, the robot can perceive the target.

Step 2: Distance Estimation. According to the images taken by eyes of the mobile robot vision system, the distance of target can be gotten depending on vision measurement technology. Of course, if the map is necessary for the robot to select its route or avoid obstacle, the robot can redraw the scene as a map. Therefore, the robot can know the position of target and get the environment information in this step.

Step 3: Motion Capture. When the target is moving, the robot can compute the motion vector of target using search algorithm. According to the target motion vector, the robot can control its drive system to follow the target. Therefore, the robot can know how to track the target.

The rest of this paper is organized as follows. Section 2 discusses the application of non-negative matrix factorization in robot navigation. The data processing and an example are demonstrated in this section. Section 3 and section 4 analyze the distance estimation and the motion estimation, respectively. Section 5 gives a model to validate the approach presented in this paper, concludes the works of this paper, also discusses some future work.

\section{TARGET RECOGNITION}

In this step, the essence of target recognition is the mechanism of computer to cognise object. Undoubtedly, the research on mechanism of the human brain to perceive the world is helpful to resolve our problem.
Some researchers found psychological and physiological evidence for parts-based representations in the brain. Consequently, certain computational theories of object recognition were presented based on such representations. According to the parts-based representations, the objects can be represented as

$$
\begin{aligned}
\text { Object }_{i}= & b_{i 1} \times \text { Part }_{1}+b_{i 2} \times \text { Part }_{2}+\cdots \\
& +b_{i j} \times \text { Part }_{j}+\cdots
\end{aligned}
$$

where

$$
b_{i j}= \begin{cases}1 & \text { if part } j \text { is present in object } i \\ 0 & \text { if part } j \text { is absent from object } i .\end{cases}
$$

Similarly, the object of image can be represented as

$$
\begin{aligned}
\text { Image }_{i}= & b_{i 1} \times \text { Feature }_{1}+b_{i 2} \times \text { Feature }_{2}+\cdots \\
& +b_{i j} \times \text { Feature }_{j}+\cdots
\end{aligned}
$$

where $b_{i j} \geq 0$ are the participation weight of feature $j$ in image $i$. Because each pixel is represented by its light intensity measured by a non-negative value, the participation weight $b_{i j}$ is inevitably a non-negative number.

The parts-based representations offer an approach to reconstruct or recognize the world. Object can be cognised based on parts perceived by human brain, and image can be recognized based on features abstracted by computer. Consequently, the hard core of our problem becomes to find a method by which computer abstracts features, or parts, from images.

D. D. Lee and H. S. Seung [3] demonstrated an algorithm for non-negative matrix factorization (NMF) that could learn parts of objects, such as parts of faces and semantic features of text. In their opinion, the image database is regarded as an $n \times m$ matrix $V$, each column of which contains $n$ non-negative pixel values of one of the $m$ facial images. Then the NMF construct approximate factorizations of the form $V \approx W H$, or

$$
V_{i \mu}=(W H)_{i \mu}=\sum_{a=1}^{r} W_{i a} H_{a \mu}
$$

The $r$ columns of $W$ are called basis images. Each column of $H$ is called an encoding and is in one-to-one correspondence with a face in $V$. An encoding consists of the coefficients by which a face is represented with a linear combination of basis images. The dimensions of the matrix factors $W$ and $H$ are $n \times r$ and $r \times m$, respectively. The rank $r$ of the factorization is generally chosen so that $(n+m) \times r<n m$, and the product $W H$ can be regarded as a compressed form of the data in $V$.

To find an approximate factorization $V \approx W H$, cost function shall be defined firstly, which quantify the quality of the approximate. Euclidean distance and Kullback-Leibler divergence, or relative entropy, are two useful measures. The cost functions based on them can be expressed as follows, respectively.

$$
\|V-W H\|^{2}=\sum_{i j}\left[V_{i j}-(W H)_{i j}\right]^{2}
$$




$$
D(V \| W H)=\sum_{i j}\left[V_{i j} \log \frac{V_{i j}}{(W H)_{i j}}-V_{i j}+(W H)_{i j}\right]
$$

The Euclidean distance $\|V-W H\|$ is non-increasing under the update rules

$$
\begin{gathered}
H_{a \mu} \leftarrow H_{a \mu} \frac{\left(W^{T} V\right)_{a \mu}}{\left(W^{T} W H\right)_{a \mu}} \\
W_{i a} \leftarrow W_{i a} \frac{\left(V H^{T}\right)_{i a}}{\left(W H H^{T}\right)_{i a}}
\end{gathered}
$$

The divergence $D(V \| W H)$ is non-increasing under the update rules

$$
\begin{aligned}
H_{a \mu} & \leftarrow H_{a \mu} \frac{\sum_{i} W_{i a} V_{i \mu} /(W H)_{i \mu}}{\sum_{k} W_{k a}} \\
W_{i a} & \leftarrow W_{i a} \frac{\sum_{\mu} H_{a \mu} V_{i \mu} /(W H)_{i \mu}}{\sum_{\nu} H_{a \nu}}
\end{aligned}
$$

The Euclidean distance and divergence are invariant under these respective updates if and only if $W$ and $H$ are at a stationary point of the distance or divergence, respectively.

D. D. Lee and H. S. Seung also gave two examples of applications of NMF on facial images and semantic analysis. In the facial images application, the grey intensities of each image were first linearly scaled so that the pixel mean and standard deviation were equal to 0.25 , and then clipped to the range $[0,1]$. NMF was performed with the iterative algorithm, starting with random initial conditions for $W$ and $H$. The algorithm was mostly converged after less than 50 iterations in their example. In fact, they have proved the convergence [4] of the NMF update rules.

According to the algorithm and application on facial images, it is obvious that the NMF can be applied to recognize the object in our problem, no matter it is face, back or something else. Because the NMF has the ability to learn the parts-based features of object, the robot can recognize target if only it has this kind of ability.

A feasible process of target recognition for our mobile tracking robot may be is

Step 1: The robot captures the image of target multiangularly, such as from head to toe, from the front to the back, from far to near, etc.

Step 2: Linear scale all these images. Construct matrix $V$ by converting each image to a column vector and combining them.

Step 3: Process the matrix $V$ by implementing NMF algorithm, and then get the basis images $W$ and encoding matrix $H$.

Step 4: For a new image matrix $U$ that includes the parts of target features or some columns of matrix $W$, new encoding matrix $J$ can be extracted by $W^{-1} U$.

Step 5: Find the part in matrix $J$ that is with the shortest distance to another part in matrix $H$. This part in Matrix $J$ contains the position information of target in matrix $U$. It means we have found the target in the new image.



(a)

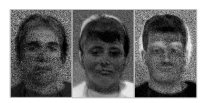

(b)

Fig. 1. Data processing by using NMF. (a) hand-aligned grey images, each of them corresponds to a column of matrix $V$; (b) the parts-based images corresponding to basis matrix $W$.

Fig. 1 shows briefly the main procedure of data processing. Firstly, the color images are converted to grey images, see Fig. 1(a). To facilitate comparison with the matrix $V$, the nine frontal views are aligned by hand, each of them corresponds to a column of matrix $V$. Fig. 1(b) shows the parts-based faces transformed by the basis image matrix $W$.

In the NMF algorithm, the rank $r$ is an important parameter that decides the dimension of characteristic subspace. The determining of $r$ shall be helpful to reduce the dimension of image matrix, but also to express target features effectively. However, there is no good way to determine it nowadays. In [5], N.D. Ho indicated that the problem of determining the non-negative rank can be solved in finite time by looping through $r=1,2, \cdots, \min (n, m)$, since the upper bound of the non-negative rank is $\min (n, m)$. Additionally, N.D. Ho et al. [6] compared the convergence speed of different algorithms, which can be a reference to the determining of $r$ value.

\section{Measure of Distance}

Binocular stereo triangulation, see Fig. 2(a), is a simple and effective approach in computational stereo [7]. It also can be regarded as a special case of two-view geometry [8]. Given the distance between aperture diaphragm $O_{L}$ and $O_{R}$, called baseline $B$, and the focal length $f$ of the cameras, object distance $D$ may be computed by similar triangles as

$$
D=\frac{f B}{x_{l}+x_{r}}=K \frac{f B}{n_{\text {pixel }}}
$$

where $x_{l}$ and $x_{r}$ are the absolute horizontal distance between image point and the left and right image center respectively, $K$ is constant, $n_{\text {pixel }}$ is displacement of pixels. Indeed binocular stereo triangulation is outdated. But if used appropriately, it will be very effective.

According to (1), the maximum estimated object distance depends on maximum focal length $f$, baseline $B$ and minimum pixel displacement $n_{\text {pixel }}$. Obviously, the maximum value is $D_{\max }=K f_{\max } B_{\max }$, where $D_{\max }, f_{\max }$ and $B_{\max }$ are maximum value of $D, f$ and $B$, respectively.

It should be noticed that the lens model in Fig. 2(a) is pinhole model. The image of the object on the image plane is top-bottom inverted and left-right inverted. And camera can revise these inversions in real picture automatically. Finally, the point on image plane lying at lower left corner may lie at upper right corner in the real picture. Therefore, these automatic revisions should be considered in measuring the 




(a)

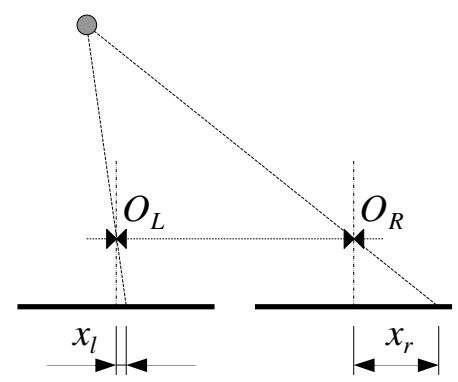

(b)

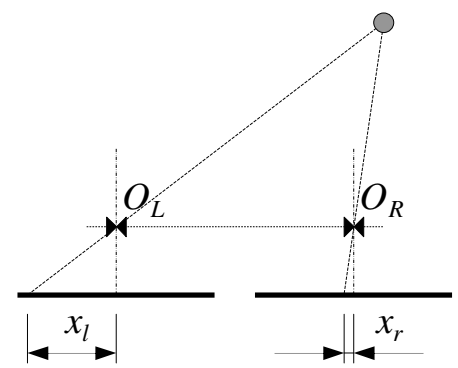

(c)

Fig. 2. Three cases of object position in image plane: the object lies (a) between two centers, (b) at the left of the left camera center, and (c) at the right of the right camera center.

distance between image point and image center in the real picture.

As shown in Fig. 2(b) and (c), if the object lies at the left of left camera or right of right camera, the object distance $D$ may be computed respectively as

$$
\begin{aligned}
& D=\frac{f B}{x_{r}-x_{l}} \\
& D=\frac{f B}{x_{l}-x_{r}}
\end{aligned}
$$

In our experiment, we select two pan-tilt-zoom webcams as the cameras of the mobile robot vision navigation system. The webcams are very cheap. In practice, it is difficult to build ideal binocular stereo system with nonverged geometry. For example, as shown in Fig. 3(a) and (b), the two optical axes of left and right camera may not parallel, or the focal lengths of two cameras may not equal, which result in two kinds of errors.

If the distance error is defined as the difference between real and measured distance, for the case shown in Fig. 3(a), the object distance error caused by unparallel optical axes,



(a)



(b)

Fig. 3. Two cases causing error of distance estimation: (a) two optical axes are unparallel, and (b) the focal lengths of two cameras are unequal.

denoted as $\operatorname{err} D_{1}$, may computed as

$$
\operatorname{err} D_{1}=\frac{f(B-f \tan \alpha)}{x_{l}+x_{r}+f \tan \alpha}-\frac{f B}{x_{l}+x_{r}}
$$

Similarly, for the case of Fig. 2(b) and (c), the object distance error caused by unparallel optical axes may computed respectively as

$$
\begin{aligned}
& \operatorname{err} D_{1}=\frac{f(B-f \tan \alpha)}{x_{r}-x_{l}+2 f \tan \alpha}-\frac{f B}{x_{r}-x_{l}} \\
& \operatorname{err} D_{1}=\frac{f(B-f \tan \alpha)}{x_{l}-x_{r}+2 f \tan \alpha}-\frac{f B}{x_{l}-x_{r}}
\end{aligned}
$$

For the case shown in Fig. 3(b), the object distance error caused by unequal focal length, denoted as $\operatorname{err} D_{2}$, may computed as

$$
\begin{aligned}
\operatorname{err} D_{2} & =\frac{f B}{x_{l}+\frac{f}{f^{\prime}} x_{r}}-\frac{f B}{x_{l}+x_{r}} \\
& =\frac{f^{\prime} f B}{f^{\prime} x_{l}+f x_{r}}-\frac{f B}{x_{l}+x_{r}}
\end{aligned}
$$

where $f^{\prime}$ is the unequal focal length of the right camera shown in $3(\mathrm{~b})$.

Similarly, for the case of Fig. 2(b) and (c), the object distance error caused by unequal focal length may computed respectively as

$$
\begin{aligned}
& \operatorname{err} D_{2}=\frac{f^{\prime} f B}{f x_{r}-f^{\prime} x_{l}}-\frac{f B}{x_{r}-x_{l}} \\
& \operatorname{err} D_{2}=\frac{f^{\prime} f B}{f x_{l}-f^{\prime} x_{r}}-\frac{f B}{x_{l}-x_{r}}
\end{aligned}
$$

According to (4), (5) and (6), the distance error caused by unparallel optical axes can be drawn, see Fig. 4. Obviously, when the object lies between two centers, the object distance error is less than that when the object lies aside. Similarly, 


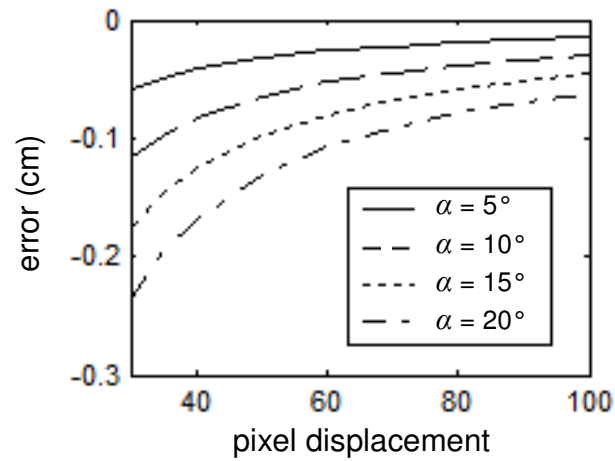

(a) corresponding error of Fig. 1(a)

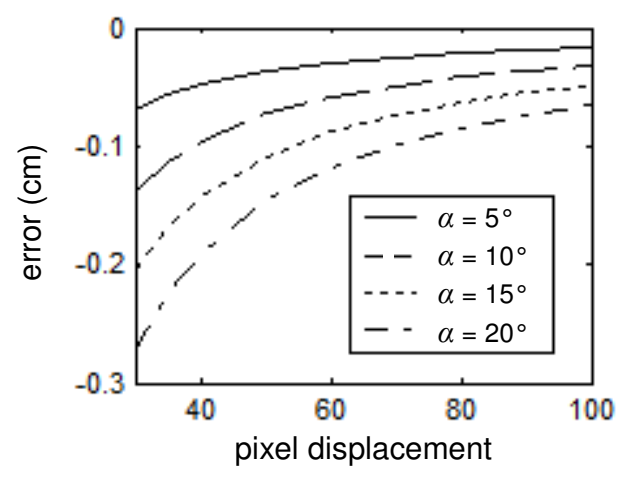

(b) corresponding error of Fig. 1(b) and (c)

Fig. 4. The object distance error caused by unparallel optical axes. Baseline is $8 \mathrm{~cm}$, and reference focal length is $4 \mathrm{~cm}$.

according to (7), (8) and (9), the distance error caused by unequal focal length can be drawn, see Fig. 5. Obviously, when the object lies between two centers, the object distance error is less than that when the object lies aside. Therefore, it is better to measure the object distance while the object lies between two centers.

Based on the error analysis above, the unparallel optical axis and unequal focal length can be corrected. Fig. 6 shows the distance curves at different baseline after correction.

Equation (1) shows that the estimation range of distance is directly proportional to $f$ and $B$. If the robot wants to adjust the distance range, it just need to increase or decrease focal length $f$ or baseline $B$. It is an effective way for the robot to observe the object at very far or very close distance. But the increase of $f$ or $B$ may also increase the object distance error, which is a matter of course, because the further the object, the larger the distance error.

\section{MOTION CAPTURE}

If the robot can compute the object's motion vector from every frame taken by its eyes, it can know the object motion, and then can track it. An effective and popular method, called block-matching motion estimation, has been widely applied in various video coding standards, such as H.261, H.263, MPEG-1, MPEG-2 and MPEG-4, and in motion-compensated video coding technique. Many fast block-matching algorithms have been developed, for example, 2-D logarithmic search,

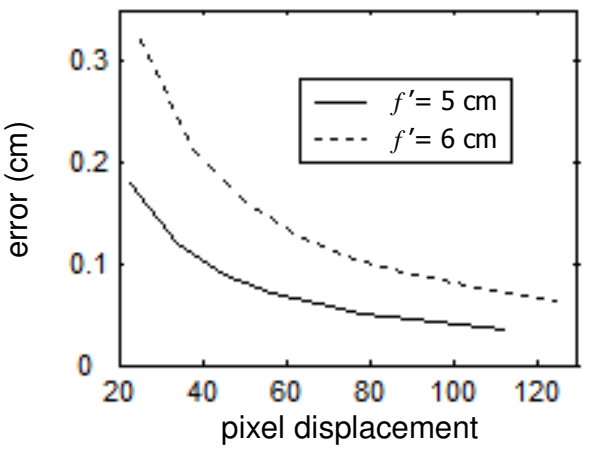

(a) corresponding error of Fig. 1(a)



(b) corresponding error of Fig. 1(b) and (c)

Fig. 5. The object distance error caused by unequal focal length. Baseline is $8 \mathrm{~cm}$, and reference focal length is $4 \mathrm{~cm}$.

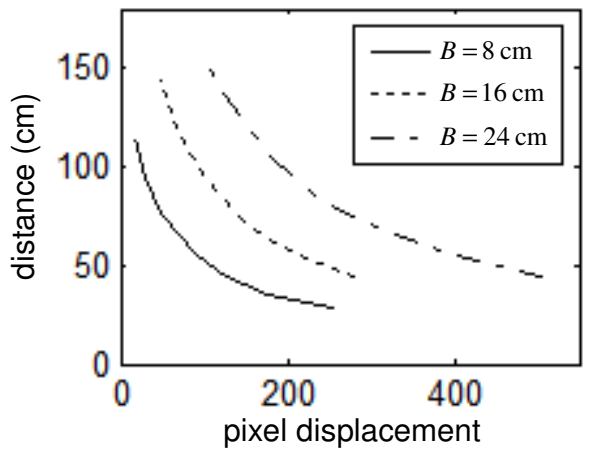

Fig. 6. The distance curves at different baseline after correcting the errors.

three-step search, conjugate direction search, cross search, new three-step search, four-step search, block-based gradient descent search, etc. These fast block-matching algorithms exploit different search patterns and search strategies for finding the optimum motion vector with drastically reduced number of search points as compared with the full search algorithm that test all the candidate blocks within the search window.

Shan Zhu and Kai Kuang Ma proposed a simple, robust and efficient fast block-matching motion estimation algorithm, called diamond search (DS) [9]. The DS algorithm employs two search patterns, called large diamond search pattern (LDSP) and small diamond search pattern (SDSP). We applied the DS algorithm to compute the object's motion vector in our experiment. 




Fig. 7. Search path example which leads to the motion vector $(-4,-2)$ in five search steps-four times of LDSP and one time SDSP at the final step. There are 24 search points in total-taking nine, five, three, three, and four search points at each step, sequentially.

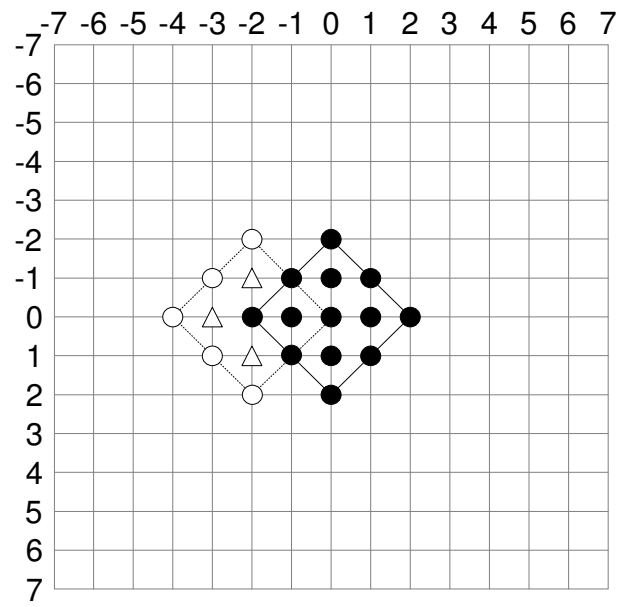

Fig. 8. Search path example which leads to the motion vector $(-2,0)$ in three search steps: once combination of LDSP and SDSP, once LDSP and once SDSP at the last step. There are 21 search points in total: taking thirteen, five and three search points at each step, sequentially.

In contrast, it is necessary to repeat briefly the DS algorithm firstly. After discussing it in detail, the improved DS algorithm used in mobile tracking robot will be introduced.

The DS algorithm is summarized as follows, as shown in Fig. 7.

Step 1: The initial LDSP is centered at the origin of the search window, and the 9 checking points of LDSP are tested. If the minimum block distortion (MBD) point calculated is located at the center position, go to Step 3; otherwise, go to Step 2.

Step 2: The MBD point found in the previous search step is re-positioned as the center point to form a new LDSP. If the new MBD point obtained is located at the center position, go to Step 3; otherwise, recursively repeat this step.

Step 3: Switch the search pattern from LDSP to SDSP. The MBD point found in this step is the final solution of the motion vector which points to the best matching block.

Firstly, the DS algorithm computes every block's motion vector; however, for mobile robot, it just need to only compute the motion vector of interested one rather than every block.

Secondly, the DS algorithm has perfect performance if the object moves to one of the 9 positions at LDSP in next frame; but if the object moves outside of LDSP or to one of the five positions at SDSP in next frame, it cannot find the object any more. Considering what the robot track is human, general movement is forward or backward, and rarely very fast left or right, so the moving outside of LDSP in next frame could be neglected. At the same time, the initial search should include LDSP and SDSP.

In our experiment, we found that the block size has important effect to the search result. The perfect situation is that the search block has the same size to the object in image. If the block is greater than the object, search is not very good.

Based on the discussion above, the improved DS algorithm could be summarized as follows, as shown in Fig. 8 .

Step 1: According on the object feature, set the block size equals to the object size. The initial search including LDSP and SDSP is centered at the origin of the search window, and the 13 checking points are tested. If the MBD point calculated is located at the center position, go to Step 3; otherwise, go to Step 2.

Step 2: The MBD point found in the previous search step is re-positioned as the center point to form a new LDSP. If the new MBD point obtained is located at the center position, go to Step 3; otherwise, recursively repeat this step.

Step 3: Switch the search pattern from LDSP to SDSP. The MBD point found in this step is the final solution of the motion vector which points to the best matching block.

Fig. 9(a) and (b) are two frames of a video. It shows the movement of a circle and a square object. In fact, the square one is the target, the method presented in this paper can estimate the object's distance and compute its motion vector, see Fig. 10.

\section{CONCLUSION}

An ease way to validate the approach of vision-based navigation system discussed in this paper is to connect webcams with computer; after receiving data from webcams and calculating the target's distance and motion vector, computer send control parameters to robot. LEGO Mindstorms NXT is a good choice for the robot. It's not necessary to fix the webcams on LEGO robot in this verification model. The final purpose of this model is to control the left and right wheel of LEGO robot rotate correctly according to the movement of target.

Fig. 11 shows the flow of the verification model. The webcams capture frames and send these data to computer, computer then recognizes target and calculates its distance and motion vector to control robot's speed and direction so as to follow the target.

In sum, the vision-based system of computer vision navigation for mobile tracking robot mentioned in this paper consists of three main parts called target recognition, distance measurement and motion capture, respectively. 


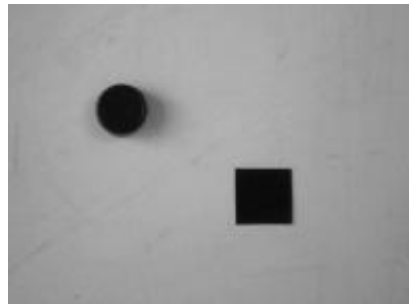

(a) the reference frame



(b) the matched frame to compute motion vector

Fig. 9. Two frames of a video. (a) is the reference frame, and (b) is the next frame. The motion vector is computed according to these adjacent frames. The black square is target. The circle one is disturbing object. Both of them are moved.

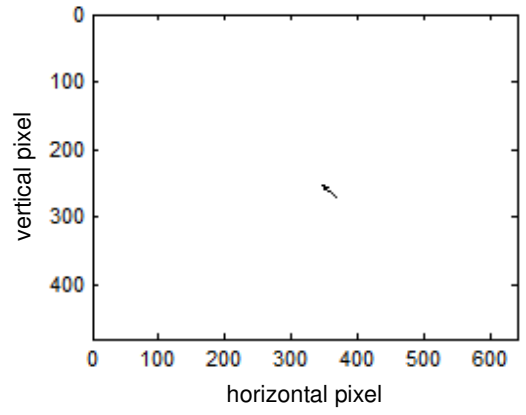

Fig. 10. The motion vector of square target. The upper left corner is the origin of image. The vector means motion vector that shows the target's displacement from start to end point.

The NMF algorithm is applied in recognition part to perceive target. Because NMF is a parts-based algorithm, it can recognize the target after learning the parts-based features. The NMF is an important development of the research on perception, which provides a feasible approach for machine to simulate the cognitive methods in human brain. However, NMF is not a perfect algorithm. For example, its localization performance of basis image is not satisfied; when it finds projecting basis vector to compress high dimensional data to low dimensional data, it ignores an important information that original data samples belong to different categories; also, there is no clear requirements of statistical relationship of the data after dimension reduction. Accordingly, many improved algorithms were developed, such as local non-negative matrix factorization (LNMF) [10], [11], sparseness non-negative matrix factorization [12], [13], [14], fisher non-negative matrix factorization (FNMF) [15], etc. It's necessary to optimize the NMF according to the requirements of mobile tracking robot; to find a method to determine the optimized rank $r$; to simplify the recognition steps.

The reasons resulting in object distance error and its cor-

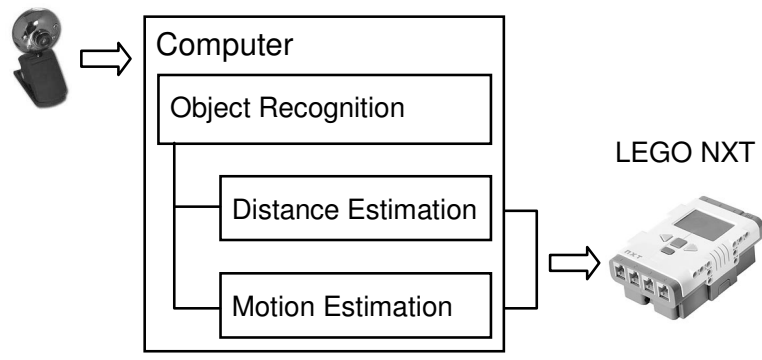

Fig. 11. The flow of the verification model.

rection methods are discussed in detail. When the object lies between two optical axes, the distance error is less than that when object lies aside. An improved DS algorithm is developed to meet the special requirement of mobile tracking robot. In future, the distance estimation model should be developed. It will be very good for the robot if it can fitting the distance curves at different focal length $f$ and baseline $B$ easily. Additionally, simpler and faster search algorithm should be developed. Of course, all of these should be realized by cheap hardware.

\section{REFERENCES}

[1] G. N. DeSouza and A. C. Kak, "Vision for mobile robot navigation: a survey," IEEE Trans. Pattern Analysis and Machine Intelligence, vol. 24, no. 2, pp. 237-267, Feb. 2002.

[2] S. Thrun, "Robotic mapping: a survey," School of Computer Science, Carnegie Mellon University, Pittsburgh, Tech. Rep., Feb. 2002.

[3] D. D. Lee and H. S. Seung, "Learning the parts of objects by nonnegative matrix factorization," Nature, vol. 401, pp. 788-791, Oct. 1999.

[4] S. Se, D. G. Lowe, and J. J. Little, "Vision-based global localization and mapping for mobile robots," IEEE Trans. on Robotics, vol. 21, no. 3, pp. 364-375, Jun. 2005.

[5] N.-D. Ho, "Non-negative matrix factorization algorithms and applications," Ph.D. dissertation, UNIVERSIT07 CATHOLIQUE DE LOUVAIN, Jun. 2008.

[6] N.-D. Ho, P. V. Dooren, and V. D. Blondel, "Descent methods for nonnegative matrix factorization," in Numerical Linear Algebra in Signals, Systems and Control, Aug. 2009, ch. 1.

[7] M. Z. Brown, D. Burschka, and G. D. Hager, "Advances in computational stereo," IEEE Trans. on Pattern Analysis and Machine Intelligence, vol. 25 , no. 8, pp. 993-1008, 2003.

[8] R. Hartley and A. Zisserman, Multiple View Geometry in Computer Vision. UK: Cambridge University Press, 2003.

[9] S. Zhu and K. K. Ma, "A new diamond search algorithm for fast blockmatching motion estimation," IEEE Trans. on Image Procesing, vol. 9, no. 2, pp. 287-290, Feb. 2000.

[10] S. Z. Li, X. Hou, H. Zhang, and Q. Cheng, "Learning spatially localized, parts-based representation," in Proc. of the 2001 IEEE Computer Society Conf. on Computer Vision and Pattern Recognition, vol. 1, 2001, pp. 207-212.

[11] T. Feng, S. Z. Li, H.-Y. Shum, and H. Zhang, "Local non-negative matrix factorization as a visual representation," in Proc. of the 2nd International Conf. on Development and Learning, Jun. 2002, pp. 178-183.

[12] W. Liu, N. Zheng, and X. Lu, "Non-negative matrix factorization for visual coding," in Proc. IEEE International Conf. on Acoustics, Speech, and Signal Processing, vol. 3, Apr. 2003, pp. 293-296.

[13] P. O. Hoyer, "Non-negative sparse coding," in Proc. Neural Networks for Signal Processing, Nov. 2002, pp. 557-565.

[14] - "Non-negative matrix factorization with sparseness constraints," Journal of Machine Learning Research, pp. 1457-1469, May 2004.

[15] Y. Wang, Y. Jia, C. Hu, and M. Turk, "Fisher non-negative matrix factorization for learning local features," in Asian Conference on Computer Vision, 2004 